\title{
TERAPIA DE REPOSIÇÃO HORMONAL NO CLIMATÉRIO: UMA REVISÃO SISTEMÁTICA
}

\section{ARTIGO DE REVISÃO}

ALVES, Letícia Furtado ${ }^{1}$, OLIVEIRA, Karine Panuce de ${ }^{2}$, SOARES, Thiago Rodrigo 3, SOUZA, Jhonata Leonardo de 4, CARVALHO, Láysa Guerra de 5, HARAGUCHI, Ana Luiza Keiko Melo ${ }^{6}$, SANTANA, Daiane Silvério ${ }^{7}$, DIAS, Camila Arimatéa Anunciação ${ }^{8}$, PATRÍCIO, Lucas Ramos ${ }^{9}$

ALVES, Letícia Furtado. Et al. Terapia de reposição hormonal no climatério: uma revisão sistemática. Revista Científica Multidisciplinar Núcleo do Conhecimento. Ano. 07, Ed. 02, Vol. 01, pp. 40-68. Fevereiro de 2022. ISSN: 2448-0959, Link de acesso: https://www.nucleodoconhecimento.com.br/saude/reposicao-hormonal

\section{RESUMO}

Normalmente as mulheres atingem a menopausa após 12 meses de amenorréia devido à falência ovariana. Nesse ínterim, ao lado da menopausa tem-se o climatério, que é um período marcado por intensa ansiedade, medo, dificuldade social e drásticas mudanças endócrinas no organismo feminino. Nessa perspectiva, surgiu a terapia de

\footnotetext{
${ }^{1}$ Ensino médio completo. Graduanda em medicina. ORCID: 0000-0003-2800-8824.

2 Ensino médio completo. Graduanda em medicina. ORCID: 0000-0002-5988-7203

${ }^{3}$ Ensino médio completo. Graduando em medicina. ORCID: 0000-0002-6466-7854.

${ }^{4}$ Ensino médio completo. Graduando em medicina. ORCID: 0000-0002-7409-2433

${ }^{5}$ Ensino médio completo. Graduanda em medicina. ORCID: 0000-0003-4385-4658.

${ }^{6}$ Ensino médio completo. Graduanda em medicina. ORCID: 0000-0002-3895-1114.

7 Ensino médio completo. Graduada em Direito. Graduanda em Medicina. ORCID: 0000-0002-4137-8996

${ }^{8}$ Ensino médio completo. Graduanda em medicina. ORCID: 0000-0002-1568-7023

${ }^{9}$ Ensino médio completo. Graduando em medicina. ORCID: 0000-0001-6598-6366
}

RC: 106172

Disponível em: https://www.nucleodoconhecimento.com.br/saude/reposicao-hormonal 
reposição hormonal (TRH), a qual representa um tratamento eficaz, com benefícios que transcendem o mero alívio da sintomatologia vasomotora. $O$ objetivo do estudo foi verificar por meio da literatura os benefícios e os riscos da TRH para alívio dos sintomas no climatério e qual a conduta do médico nesse contexto. Para isto, adotouse como metodologia a revisão sistemática da literatura, construída através da consulta às bases de dados e outros trabalhos relacionados ao tema, nos meses de julho de 2021 a janeiro de 2022, respondendo à questão norteadora: Quais são as vantagens e desvantagens da TRH no climatério e qual o papel do médico nesse contexto? Portanto, trata-se de uma revisão fundamentada nas bases de dados SciELO, Web of Science e PubMed. Utilizaram-se artigos nas línguas portuguesa e inglesa. Os descritores foram os presentes no Mesh/Decs e operadores booleanos, "AND" e "OR". Foram incluídos 33 trabalhos escritos entre 2003 e 2021. O artigo visa esclarecer que os benefícios da TRH superam os malefícios para as mulheres no climatério, desde que não haja contraindicação à reposição hormonal. Os principais resultados evidenciaram que a TRH atua na melhora dos sintomas vasomotores e urogenitais, na diminuição do risco de diabetes, na prevenção da osteoporose, evitando doenças cardiovasculares, na promoção da saúde mental e na diminuição do risco de câncer de endométrio. Todavia, a TRH pode aumentar o risco de doença tromboembólica, de AVE, de câncer de mama e de ovário. Em suma, é importante destacar que a terapia de reposição hormonal na fase climatérica é um tratamento eficaz diante de um acompanhamento médico e mediante a uma implementação da promoção de saúde da mulher para a obtenção de uma boa qualidade de vida. Porém, é fundamental ressaltar que o uso de TRH sem a orientação de um profissional adequado poderá trazer efeitos indesejados.

Palavras-chave: Reposição hormonal, Saúde da mulher, Climatério, Menopausa.

\section{INTRODUÇÃO}

As políticas em saúde, antigamente restritas à função reprodutiva e à anatomia da mulher, evoluíram no sentido de uma atenção integral à saúde do sexo feminino

RC: 106172

Disponível em: https://www.nucleodoconhecimento.com.br/saude/reposicao-hormonal 
(FERREIRA et al., 2020). Dessa maneira, uma das etapas da vida da mulher que merece destaque e um atendimento mais humanizado é a fase do climatério e/ou menopausa. Na maioria dos países desenvolvidos, $10 \%$ da população tem mais de 50 anos e 95\% das mulheres alcançam a menopausa, que é a última menstruação confirmada depois de 12 meses de amenorréia devido à falência ovariana (MARTINS et al., 2009).

Ao lado da menopausa tem-se o climatério, o qual é a fase da vida da mulher antes e depois da menopausa. Portanto, o climatério é transição entre a fase reprodutiva e a não reprodutiva da vida da mulher com sintomas característicos devido a diminuição dos hormônios femininos. O climatério traz complicações sociais e concernentes ao processo de senescência, um período marcado por ansiedade, diversos receios e dificuldade social, decorrente das inúmeras sintomatologias apresentadas (FERREIRA et al., 2020).

Nesse ínterim, o objetivo desse estudo foi constatar através da literatura os benefícios e os riscos da TRH para alívio dos sintomas no climatério e qual a conduta do médico nesse contexto. E a questão norteadora usada para delinear o estudo e atingir o objetivo foi a seguinte: Quais são as vantagens e desvantagens da TRH no climatério e qual o papel do médico nesse contexto? Os sintomas climatéricos afetam entre 60 a $80 \%$ das mulheres e repercutem em desconforto físico e psíquico que aumentam com a severidade dos sintomas. Dependendo da intensidade e da frequência dos sintomas, especialmente das ondas de calor, o bem-estar e a qualidade de vida da paciente podem ser drasticamente afetados (MARTINS et al., 2009).

Nessa perspectiva, os sintomas ocorrem em decorrência da diminuição da progesterona e do estrogênio originados da conversão periférica da testosterona e androstenediona em estrona. Além do aludido, na maioria das vezes as pacientes climatéricas também apresentam concentrações menores de androgênios. Assim, as consequências da diminuição da progesterona, do estrogênio e dos androgênios são: um período de instabilidade vasomotora, atrofia dos caracteres sexuais secundários, 
diminuição da lubrificação vaginal, sarcopenia, aumento da atividade osteoclástica, tendência à depressão e aumento do risco de doenças cardiovasculares (VALADARES et al., 2008).

Nessa conjuntura, surgiu a terapia de reposição hormonal (TRH), que tem sido objeto de muita discussão desde a década de 1960, período no qual os médicos costumavam prescrever estrogenioterapia isolada para todas as pacientes menopausadas, originando corolários principalmente em nível endometrial e mamário (maior risco de câncer de mama). Contudo, em 1980, os médicos notaram o efeito protetor das progestinas no endométrio e o uso da progesterona combinada com o estrogênio obteve ascensão (PARDINI, 2014).

Destarte, a terapia hormonal é um tratamento eficaz, com vantagens que transcendem o mero alívio da sintomatologia vasomotora (ALMEIDA et al., 2014). A sintomatologia desse período repercute em mudanças psicossociais, hormonais e físicas. As quais podem ser manejados através das terapias que conciliam progestinas, estrogênicos e/ou testosteronas, como, por exemplo, a prevenção do declínio cognitivo, da osteoporose, da sarcopenia, da instabilidade de humor, da atrofia de caracteres sexuais secundários (decorrência da queda androgênica), das doenças crônicodegenerativas, da aterosclerose e da doença de alzheimer, dentre os diversos prejuízos que o hipogonadismohipogonadotrófico fisiológico pode trazer a saúde feminina (BEZERRA et al., 2019; ALMEIDA et al., 2014).

Por outro lado, há uma série de fatores a serem previamente analisados e que podem colocar a mulher em um panorama onde não haverá possibilidade de realização da TRH devido a situações de risco (BEZERRA et al., 2019). 


\section{DESENVOLVIMENTO}

\subsection{METODOLOGIA}

O presente estudo trata-se de uma revisão sistemática da literatura construída através da consulta às bases de dados e outros trabalhos relacionados ao tema, nos meses de julho de 2021 a janeiro de 2022. Diante disso, vale salientar que o pedido de pesquisa não é prático, assim, não foi necessário passar pelo Comitê de Ética em Pesquisa (CEP).

Esta revisão consiste em um método de pesquisa no qual utiliza-se a prática baseada em evidências. Sendo esta imprescindível no âmbito da saúde, uma vez que, há uma síntese das pesquisas disponíveis de uma temática em questão e permite responder à questão norteadora do presente estudo: Quais são as vantagens e desvantagens da TRH no climatério e qual o papel do médico nesse contexto?

Esta revisão foi fundamentada nas bases de dados SciELO, Web of Science e PubMed. Utilizou-se artigos nas línguas portuguesa e inglesa. Os descritores foram os presentes no Mesh/Decs e operadores booleanos, "AND" e "OR". Utilizou-se as seguintes palavras-chave: "Reposição hormonal", "Saúde da mulher", "Menopausa", "Climatério". Foram excluídos os estudos fora do período escolhido para a pesquisa, estudos em outros idiomas, artigos que não contemplavam o tema proposto ou os descritores selecionados.

Dessa forma, baseado no modelo de busca acima, foram encontrados um total de 1.100 artigos nas bases de dados consultadas. Porém, apenas 33 trabalhos, escritos entre 2003 e 2021, atenderam aos critérios de inclusão.

Nesse contexto, tanto a análise quanto a síntese dos dados compilados nos artigos incluídos foram retratados de forma descritiva. Isso foi possível, por meio da construção de uma tabela contendo as principais informações dos artigos que foram selecionados na pesquisa, sendo essas: título, autores, ano de publicação,

RC: 106172

Disponível em: https://www.nucleodoconhecimento.com.br/saude/reposicao-hormonal 
metodologia, os principais resultados e discussão, com a finalidade de reunir o conhecimento engendrando no concerne ao tema explorado na revisão.

\subsection{RESULTADOS}

\section{Características dos Estudos}

Nesta revisão sistemática foram incluídos 33 estudos associados às vantagens e desvantagens da TRH no climatério e ao papel do médico nesse contexto. Dentre eles, encontram-se 24 revisões de literatura (ALVES et al., 2021; ANAGNOSTIS et al., 2020; ARAÚJO JÚNIOR; ATHANAZIO., 2007; BEZERRA et al., 2019; BILLECI et al., 2008; BORGES el al., 2015; CAMPIOLO et al., 2003; DE FRANCISCIS et al., 2019; FERREIRA et al., 2020; GAMBACCIANI et al., 2019; GOSSET et al., 2021; LANGER et al., 2021; LA VECCHIA., 2017; LIU et al., 2019; NAFTOLIN et al., 2019; OLIVEIRA et al., 2019; PACIUC, 2020; PALMISANO et al., 2017; PARDINI, 2014; POLONINI et al., 2011; RAGLAN et al., 2019; RANTANEN; TATLISUMAK., 2013; YOO et al., 2020; ROZENBERG et al., 2021), 1 estudo observacional descritivo do tipo coorte (FERNANDES et al., 2021), 2 estudos observacionais descritivos do tipo caso-controle (ALMEIDA et al., 2011; VINOGRADOVA et al., 2020), 3 estudos observacionais descritivos do tipo transversal (FERNANDES; ROZENTHAL, 2008; MARTINS et al., 2009; ROBAINA et al., 2015), 1 estudo qualitativo exploratório (FERREIRA et al., 2013), 1 pesquisa epidemiológica, prospectiva e longitudinal (MIRANDA et al., 2014) e 1 análise secundária de dados de um estudo populacional de corte transversal (VALADARES et al., 2008). A Tabela 1 contém informações resumidas sobre cada um dos estudos.

Análise dos Resultados

Os principais resultados evidenciaram que a TRH atua na melhora dos sintomas vasomotores e urogenitais, na diminuição do risco de diabetes e intolerância a hidratos de carbono, na prevenção da osteoporose, evitando doenças cardiovasculares, na promoção da saúde mental e na diminuição do risco de câncer endometrial. No

RC: 106172

Disponível em: https://www.nucleodoconhecimento.com.br/saude/reposicao-hormonal 
entanto, a TRH pode aumentar o risco de doença tromboembólica, de AVE, de câncer de mama e de ovário. Os resultados também esclareceram a importância do papel do médico nesse período climatérico.

Tabela 1: Descrições dos estudos selecionados nesta revisão da literatura.

\begin{tabular}{|c|c|c|}
\hline Título & $\begin{array}{l}\text { Autores, ano de } \\
\text { publicação, } \\
\text { metodologia }\end{array}$ & $\begin{array}{l}\text { Principais resultados } \\
\text { discussão }\end{array}$ \\
\hline $\begin{array}{l}\text { Impacto da terapia } \\
\text { hormonal sobre o peso } \\
\text { corpóreo. }\end{array}$ & $\begin{array}{l}\text { (ALMEIDA et al., } \\
2011) \\
\text { Estudo } \\
\text { observacional } \\
\text { descritivo do tipo } \\
\text { caso-controle. }\end{array}$ & $\begin{array}{l}\text { Constatou-se um aumento } \\
\text { ponderal médio superior no grupo } \\
\text { controle (sem TRH) relativamente } \\
\text { ao grupo de mulheres sob TRH } \\
\text { (434 vs } 76 \mathrm{~g} \text { ), embora a diferença } \\
\text { verificada não significativa } \\
\text { estatisticamente a } \\
\text { ( } \mathrm{p}=0,406) \text {. Ressaltou a } \\
\text { importância do papel do médico } \\
\text { em orientar a paciente quanto a } \\
\text { melhora dos hábitos de vida para } \\
\text { amenizar o ganho de peso e outras } \\
\text { repercussões da menopausa. }\end{array}$ \\
\hline $\begin{array}{l}\text { Osteoporose: } \\
\text { fisiopatologia das } \\
\text { alterações histológicas } \\
\text { do tecido ósseo e } \\
\text { prevenção com } \\
\text { atividade física. }\end{array}$ & $\begin{array}{l}\text { (ALVES et al., 2021) } \\
\text { Revisão sistemática } \\
\text { da literatura. }\end{array}$ & $\begin{array}{l}\text { Salientou as alterações } \\
\text { histológicas do osso na } \\
\text { osteoporose e a ação da atividade } \\
\text { física e do estrogênio como } \\
\text { protetores do tecido ósseo. }\end{array}$ \\
\hline $\begin{array}{l}\text { Estrogen and bones } \\
\text { after menopause: a }\end{array}$ & $\begin{array}{l}\text { (ANAGNOSTIS et } \\
\text { al., } 2020)\end{array}$ & $\begin{array}{l}\text { TRH na menopausa é eficaz na } \\
\text { prevenção da perda óssea } \\
\text { associada à menopausa e na }\end{array}$ \\
\hline
\end{tabular}

RC: 106172

Disponível em: https://www.nucleodoconhecimento.com.br/saude/reposicao-hormonal 


\begin{tabular}{|c|c|c|}
\hline $\begin{array}{l}\text { reappraisal of data and } \\
\text { future perspectives. }\end{array}$ & $\begin{array}{l}\text { Revisão } \\
\text { literatura. }\end{array}$ & $\begin{array}{l}\text { diminuição do risco de fratura } \\
\text { vertebral, não vertebral e de } \\
\text { quadril. }\end{array}$ \\
\hline $\begin{array}{l}\text { Terapia de reposição } \\
\text { hormonal e o câncer do } \\
\text { endométrio. }\end{array}$ & $\begin{array}{l}\text { (ARAÚJO JÚNIOR; } \\
\text { ATHANAZIO., } \\
\text { 2007) } \\
\text { Revisão da } \\
\text { literatura. }\end{array}$ & $\begin{array}{l}\text { O trabalho evidenciou o aumento } \\
\text { do risco de câncer de mama e a } \\
\text { diminuição do risco de câncer de } \\
\text { endométrio em mulheres que } \\
\text { faziam o uso da TRH combinada. }\end{array}$ \\
\hline $\begin{array}{l}\text { Terapia de reposição } \\
\text { hormonal na } \\
\text { menopausa. }\end{array}$ & $\begin{array}{l}\text { (BEZERRA et al., } \\
2019) \\
\text { Revisão } \\
\text { bibliográfica } \\
\text { sistemática. }\end{array}$ & $\begin{array}{l}\text { O aumento do risco de câncer de } \\
\text { mama e doença tromboembólica, } \\
\text { que podem ser efeitos adversos da } \\
\text { TRH no climatério, é baixo no } \\
\text { primeiro ano de tratamento. E a } \\
\text { TRH é indicada para tratar e } \\
\text { amenizar os sintomas decorrentes } \\
\text { da menopausa, visando beneficiar } \\
\text { a qualidade de vida das pacientes } \\
\text { que fazem o uso do tratamento. }\end{array}$ \\
\hline $\begin{array}{l}\text { Hormone replacement } \\
\text { therapy and stroke. }\end{array}$ & $\begin{array}{l}\text { (BILLECl et al., } \\
\text { 2008) } \\
\text { Revisão } \\
\text { literatura. }\end{array}$ & $\begin{array}{l}\text { Embora a TRH tenha sido } \\
\text { associada à redução do risco de } \\
\text { doença cardíaca em estudos } \\
\text { observacionais, os resultados } \\
\text { sobre o acidente vascular cerebral } \\
\text { têm sido menos claros. } \\
\text { Recentemente, evidências } \\
\text { mostraram que a TRH não reduz, } \\
\text { mas na verdade aumenta o risco } \\
\text { de AVE. }\end{array}$ \\
\hline
\end{tabular}




\begin{tabular}{|c|c|c|}
\hline $\begin{array}{l}\text { Terapia com Hormônios } \\
\text { Sexuais Femininos e } \\
\text { Fenômenos } \\
\text { Tromboembólicos: uma } \\
\text { Revisão de Literatura. }\end{array}$ & $\begin{array}{l}\text { (BORGES el al., } \\
\text { 2015) } \\
\text { Revisão } \\
\text { literatura. }\end{array}$ & $\begin{array}{l}\text { Aumento do risco de fenômenos } \\
\text { tromboembólicos por uso da } \mathrm{TRH} \text {. }\end{array}$ \\
\hline $\begin{array}{l}\text { Tromboembolismo } \\
\text { venoso e terapia de } \\
\text { reposição hormonal da } \\
\text { menopausa: uma } \\
\text { análise clínico- } \\
\text { epidemiológica. }\end{array}$ & $\begin{array}{l}\text { (CAMPIOLO et al., } \\
\text { 2003) } \\
\text { Revisão de } \\
\text { literatura. }\end{array}$ & $\begin{array}{l}\text { Recentes estudos observacionais } \\
\text { têm indicado possível aumento no } \\
\text { risco de tromboembolismo venoso } \\
\text { com a TRH. Essas observações } \\
\text { levaram à suspeita de que } \\
\text { estrogênios exógenos, como os } \\
\text { usados na TRH, poderiam elevar o } \\
\text { risco de tromboembolismo. Este } \\
\text { risco de tromboembolismo com a } \\
\text { TRH parece ser bem maior em } \\
\text { mulheres com história pessoal ou } \\
\text { familiar de trombose venosa } \\
\text { profunda ou embolismo pulmonar, } \\
\text { assim como presença de severas } \\
\text { veias varicosas, obesidade, } \\
\text { cirurgia, trauma, câncer, ou } \\
\text { permanência no leito por longo } \\
\text { período. }\end{array}$ \\
\hline Nutraceutical & (DE FRANCISCIS & O médico deve incluir na discussão \\
\hline $\begin{array}{l}\text { Approach } \\
\text { Menopausal } \\
\text { Complaints. }\end{array}$ & $\begin{array}{lr}\text { et al., } & \text { 2019) } \\
\text { Revisão } & \text { da } \\
\text { literatura. } & \end{array}$ & $\begin{array}{l}\text { com suas pacientes todas as } \\
\text { abordagens disponíveis para alívio } \\
\text { dos sintomas do climatério, } \\
\text { concedendo todas as informações } \\
\text { necessárias para uma escolha } \\
\text { consciente e compartilhada. }\end{array}$ \\
\hline
\end{tabular}




\begin{tabular}{|c|c|c|}
\hline $\begin{array}{l}\text { ndependência } \\
\text { uncional após acidente } \\
\text { lascular cerebral (AVC) } \\
\text { squêmico em relação à } \\
\text { isiopatologia de acordo } \\
\text { com TOAST. }\end{array}$ & $\begin{array}{l}\text { (FERNANDES et } \\
\text { al., 2021) } \\
\text { Estudo } \\
\text { observacional } \\
\text { descritivo do tipo } \\
\text { coorte. }\end{array}$ & $\begin{array}{l}\text { Etiologia, epidemiologia e } \\
\text { fisiopatologia do AVC. Um dos } \\
\text { fatores de risco para o } \\
\text { desenvolvimento do AVC } \\
\text { isquêmico é o uso da TRH. }\end{array}$ \\
\hline $\begin{array}{l}\text { valiação da } \\
\text { intomatologia } \\
\text { epressiva de mulheres } \\
\text { o climatério com a } \\
\text { scala de rastreamento } \\
\text { opulacional para } \\
\text { epressão CES-D. }\end{array}$ & $\begin{array}{l}\text { (FERNANDES; } \\
\text { ROZENTHAL., } \\
\text { 2008) } \\
\text { Estudo } \\
\text { observacional } \\
\text { descritivo do tipo } \\
\text { transversal. }\end{array}$ & $\begin{array}{l}\text { Aumento dos sintomas } \\
\text { depressivos no climatério devido a } \\
\text { oscilação hormonal e a importância } \\
\text { da TRH. }\end{array}$ \\
\hline $\begin{array}{l}\text { Saúde da Mulher, } \\
\text { Gênero, } \\
\text { Públíticas e Educação } \\
\text { Médica: agravos no } \\
\text { contexto de pandemia. }\end{array}$ & $\begin{array}{l}\text { (FERREIRA et al., } \\
\text { 2020) } \\
\text { Revisão narrativa } \\
\text { da literatura. }\end{array}$ & $\begin{array}{l}\text { Saúde da mulher e as } \\
\text { repercussões do climatério. Uma } \\
\text { nova visão em relação a essa fase } \\
\text { delicada da vida da mulher. }\end{array}$ \\
\hline $\begin{array}{l}\text { Menopausa: marco } \\
\text { biopsicossocial } \quad \text { do } \\
\text { envelhecimento } \\
\text { feminino. }\end{array}$ & $\begin{array}{l}\text { (FERREIRA et al., } \\
2013) \\
\text { Estudo qualitativo } \\
\text { exploratório }\end{array}$ & $\begin{array}{l}\text { Relação da menopausa e/ou } \\
\text { climatério com as alterações } \\
\text { biopsicossociais engendradas por } \\
\text { esta fase da vida feminina. }\end{array}$ \\
\hline $\begin{array}{l}\text { Hormone replacement } \\
\text { herapy and prevention } \\
\text { of chronic conditions. }\end{array}$ & $\begin{array}{lr}\text { (GAMBACCIANI } & \text { et } \\
\text { al., } & \text { 2019) } \\
\text { Revisão } & \text { da } \\
\text { literatura. } & \end{array}$ & $\begin{array}{l}\text { Os sintomas do climatério estão } \\
\text { relacionados a um risco } \\
\text { aumentado de doenças crônicas, } \\
\text { incluindo hipertensão e doenças } \\
\text { cardiovasculares. E a TRH pode } \\
\text { atenuar tais riscos de acordo com } \\
\text { evidências científicas de estudos } \\
\text { pré-clínicos, clínicos, }\end{array}$ \\
\hline
\end{tabular}




\begin{tabular}{|c|c|c|}
\hline & & $\begin{array}{l}\text { epidemiológicos e também } \\
\text { randomizados. }\end{array}$ \\
\hline $\begin{array}{l}\text { Menopausal hormone } \\
\text { therapy for the } \\
\text { management of } \\
\text { osteoporosis. }\end{array}$ & 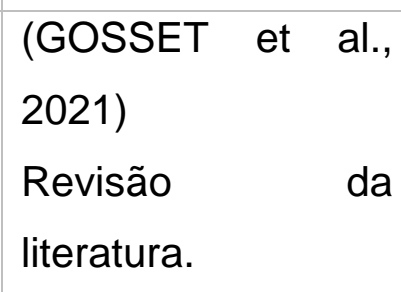 & $\begin{array}{l}\text { A importância da TRH para } \\
\text { prevenir a perda óssea e a } \\
\text { degradação da microarquitetura } \\
\text { óssea. }\end{array}$ \\
\hline $\begin{array}{l}\text { Hormone replacement } \\
\text { therapy - where are we } \\
\text { now? }\end{array}$ &  & $\begin{array}{l}\text { A TRH reduz a mortalidade por } \\
\text { todas as causas e os riscos de } \\
\text { doença coronariana, osteoporose } \\
\text { e demências. }\end{array}$ \\
\hline $\begin{array}{l}\text { Ovarian cancer: } \\
\text { epidemiology and risk } \\
\text { factors. }\end{array}$ & $\begin{array}{l}\text { (LA VECCHIA., } \\
2017) \\
\text { Revisão } \\
\text { literatura. }\end{array}$ & $\begin{array}{l}\text { O estudo traz uma visão geral da } \\
\text { epidemiologia do câncer de ovário } \\
\text { e resume os principais resultados } \\
\text { de uma rede de estudos de caso- } \\
\text { controle na Itália e do Grupo } \\
\text { Colaborativo em Estudos } \\
\text { Epidemiológicos do Câncer de } \\
\text { Ovário. E um dos principais fatores } \\
\text { de risco para o desenvolvimento do } \\
\text { câncer ovariano foi o uso da TRH. }\end{array}$ \\
\hline $\begin{array}{l}\text { Menopausal Hormone } \\
\text { Replacement Therapy } \\
\text { and the Risk of Ovarian } \\
\text { Cancer: A Meta- } \\
\text { Analysis. }\end{array}$ & $\begin{array}{l}\text { (LIU et al., 2019) } \\
\text { Meta-análise. }\end{array}$ & $\begin{array}{l}\text { Esta meta-análise sugere que a } \\
\text { TRH na menopausa pode } \\
\text { aumentar o risco de câncer de } \\
\text { ovário, especialmente para } \\
\text { tumores serosos e endometrióides. }\end{array}$ \\
\hline $\begin{array}{l}\text { Qualidade de vida em } \\
\text { mulheres na pós- } \\
\text { menopausa, usuárias e } \\
\text { não usuárias de terapia } \\
\text { hormonal. }\end{array}$ & $\begin{array}{lr}\text { (MARTINS et al., } \\
\text { 2009) } & \\
\text { Estudo } & \text { clínico } \\
\text { analítico } & \text { e } \\
\text { transversal. } & \end{array}$ & $\begin{array}{l}\text { O estudo fez uma avaliação dos } \\
\text { sintomas climatéricos e utilizou o } \\
\text { Índice Menopausal de Blatt e } \\
\text { Kupperman (IMBK). Foram } \\
\text { avaliados os seguintes sintomas: }\end{array}$ \\
\hline
\end{tabular}




\begin{tabular}{|c|c|c|}
\hline & & $\begin{array}{l}\text { fogachos, insônia, parestesia, } \\
\text { nervosismo, melancolia, vertigem, } \\
\text { artralgia/ mialgia, cefaléia, } \\
\text { palpitação, zumbido, função } \\
\text { sexual, déficit cognitivos } \\
\text { e diminuição da auto-estima. A } \\
\text { intensidade de cada sintoma } \\
\text { também foi analisada no estudo e } \\
\text { a melhora sintomatológica foi } \\
\text { verificada com uso da TRH. }\end{array}$ \\
\hline $\begin{array}{l}\text { Qualidade de vida em } \\
\text { mulheres no climatério } \\
\text { atendidas na Atenção } \\
\text { Primária }\end{array}$ & $\begin{array}{l}\text { (MIRANDA et al., } \\
\text { 2014). } \\
\text { Pesquisa } \\
\text { epidemiológica, } \\
\text { prospectiva e } \\
\text { longitudinal }\end{array}$ & $\begin{array}{l}\mathrm{Na} \text { presente pesquisa, as } \\
\text { participantes eram usuárias da } \\
\text { atenção básica e, portanto, } \\
\text { pertencentes a estratos sociais } \\
\text { menos favorecidos e de baixa } \\
\text { escolaridade, em sua maioria. A } \\
\text { TRH tem efeitos significativos na } \\
\text { amenização dos fenômenos } \\
\text { vasomotores, o que pode ser } \\
\text { observado na comparação } \\
\text { intragrupo intervenção. }\end{array}$ \\
\hline $\begin{array}{l}\text { Cardiovascular health } \\
\text { and the menopausal } \\
\text { woman: the role of } \\
\text { estrogen and when to } \\
\text { begin and end hormone } \\
\text { treatment }\end{array}$ & $\begin{array}{l}\text { (NAFTOLIN et al., } \\
\text { 2019) } \\
\text { Revisão } \\
\text { literatura. }\end{array}$ & $\begin{array}{l}\text { Atualmente, as mulheres em } \\
\text { menopausa precoce recebem } \\
\text { tratamento com estrogênio (TE) } \\
\text { até que haja evidência de } \\
\text { complicações. Mulheres normais } \\
\text { que iniciaram o tratamento } \\
\text { hormonal da menopausa que } \\
\text { recebem tratamento por décadas } \\
\text { sem complicações relacionadas a }\end{array}$ \\
\hline
\end{tabular}




\begin{tabular}{|c|c|c|}
\hline & & $\begin{array}{l}\text { hormônios foram relatadas, e os } \\
\text { estudos de acompanhamento WHI } \\
\text { são promissores de cardioproteção } \\
\text { a longo prazo pós-tratamento. }\end{array}$ \\
\hline $\begin{array}{l}\text { Terapia de reposição } \\
\text { hormonal: uma análise } \\
\text { dos benefícios } \\
\text { malefícios no manejo de } \\
\text { mulheres climatéricas e } \\
\text { menopausadas }\end{array}$ & $\begin{array}{l}\text { (OLIVEIRA et al., } \\
\text { 2019) } \\
\text { Revisão } \\
\text { literatura. }\end{array}$ & $\begin{array}{l}\text { Diante de uma indicação adequada } \\
\text { de } \mathrm{TRH} \text {, por meio da análise do } \\
\text { perfil da mulher e de seus } \\
\text { antecedentes fisiológicos } \\
\text { patológicos, sua implementação é } \\
\text { majoritariamente benéfica na } \\
\text { prevenção de diversas afecções, } \\
\text { sendo, atualmente, uma via } \\
\text { terapêutica satisfatória e que, por } \\
\text { meio de suas limitações, vem } \\
\text { estimulando o desenvolvimento de } \\
\text { novas tecnologias que desejam } \\
\text { tornar a falência ovariana e seu } \\
\text { consequente impacto na produção } \\
\text { hormonal feminina, a menos } \\
\text { danosa possível. }\end{array}$ \\
\hline $\begin{array}{l}\text { Hormone Therapy in } \\
\text { Menopause }\end{array}$ & $\begin{array}{lr}\text { (PACIUC, } & \text { 2020). } \\
\text { Revisão } & \text { de } \\
\text { literatura. } & \end{array}$ & $\begin{array}{l}\text { Sintomas vulvovaginais e urinários } \\
\text { locais, agora denominados } \\
\text { síndrome geniturinária da } \\
\text { menopausa (GSM), são } \\
\text { observados em } 50 \% \text { das mulheres } \\
\text { na pós-menopausa e impactam } \\
\text { negativamente na qualidade de } \\
\text { vida. O estrogênio continua sendo } \\
\text { o tratamento mais eficaz para } \\
\text { sintomas vasomotores e GSM, }\end{array}$ \\
\hline
\end{tabular}

RC: 106172

Disponível em: https://www.nucleodoconhecimento.com.br/saude/reposicao-hormonal 


\begin{tabular}{|c|c|c|}
\hline & & $\begin{array}{l}\text { para prevenção de osteoporose e } \\
\text { alívio de sintomas, bem como } \\
\text { prevenção de doenças crônicas } \\
\text { em mulheres que experimentam } \\
\text { menopausa prematura, seja por } \\
\text { insuficiência ovariana primária } \\
\text { (IOP) ou etiologias iatrogênicas. }\end{array}$ \\
\hline $\begin{array}{l}\text { Estrogens in the } \\
\text { Regulation of Liver Lipid } \\
\text { Metabolism }\end{array}$ & $\begin{array}{l}\text { (PALMISANO et al., } \\
\text { 2017). } \\
\text { Revisão de } \\
\text { literatura. }\end{array}$ & $\begin{array}{l}\text { O tratamento de mulheres na pós- } \\
\text { menopausa com várias } \\
\text { formulações de estrogênio não } \\
\text { restaurar a proteção contra } \\
\text { doenças cardiovasculares } \\
\text { observada em mulheres na pré- } \\
\text { menopausa. Estrogênio terapias e } \\
\text { abordagens de tratamento } \\
\text { hormonal podem proteger contra } \\
\text { fígado gorduroso, resistência à } \\
\text { insulina e diabetes, mas não } \\
\text { protegem de forma conclusiva das } \\
\text { doenças cardiovasculares. }\end{array}$ \\
\hline $\begin{array}{lr}\text { Terapia de reposição } \\
\text { hormonal } \\
\text { menopausa }\end{array}$ & $\begin{array}{lr}\text { (PARDINI, } & \text { 2014). } \\
\text { Revisão } & \text { de } \\
\text { literatura. } & \end{array}$ & $\begin{array}{l}\text { Estudos observacionais dos efeitos } \\
\text { metabólicos e vasculares do } \\
\text { estrógeno sugerem um benefício } \\
\text { em potencial na redução do risco } \\
\text { de doenças vasculares, mas } \\
\text { estudos randomizados } \\
\text { controlados não demonstraram } \\
\text { nenhuma evidência de que a } \\
\text { terapia hormonal pudesse } \\
\text { beneficiar as mulheres com }\end{array}$ \\
\hline
\end{tabular}

RC: 106172

Disponível em: https://www.nucleodoconhecimento.com.br/saude/reposicao-hormonal 


\begin{tabular}{|c|c|c|}
\hline & & $\begin{array}{l}\text { doença vascular previamente } \\
\text { instalada ou em mulheres } \\
\text { aparentemente saudáveis. } \\
\text { aumento do risco de câncer de } \\
\text { mama e doença tromboembólica } \\
\text { tem se confirmado nesses estudos. }\end{array}$ \\
\hline $\begin{array}{l}\text { A terapia de reposição } \\
\text { hormonal e a saúde da } \\
\text { mulher no climatério: } \\
\text { riscos e benefícios. }\end{array}$ & $\begin{array}{l}\text { (POLONINI et al., } \\
\text { 2011). } \\
\text { Revisão } \\
\text { literatura. }\end{array}$ & $\begin{array}{l}\text { A Terapia de Reposição Hormonal } \\
\text { é um tratamento importante para } \\
\text { mulheres que sofrem de sintomas } \\
\text { mais graves advindos da fase do } \\
\text { climatério. Porém, deve ser feito } \\
\text { um acompanhamento médico } \\
\text { rigoroso durante todo o período, } \\
\text { visto que a terapia estrogênica } \\
\text { pode acarretar vários efeitos } \\
\text { colaterais severos. O período da } \\
\text { terapia também deve ser } \\
\text { delimitado em cinco anos, período } \\
\text { este considerado máximo pelo } \\
\text { FDA. }\end{array}$ \\
\hline $\begin{array}{l}\text { Depression during } \\
\text { perimenopause: the role } \\
\text { of the obstetrician- } \\
\text { gynecologist }\end{array}$ & $\begin{array}{l}\text { (RAGLAN et al., } \\
\text { 2019). } \\
\text { Revisão narrativa. }\end{array}$ & $\begin{array}{l}\text { Os sintomas de depressão podem } \\
\text { ser diferentes em mulheres na } \\
\text { perimenopausa em comparação } \\
\text { com mulheres mais jovens ou mais } \\
\text { velhas, e muitas vezes são } \\
\text { descartados como parte da } \\
\text { menopausa normal. Os } \\
\text { profissionais que cuidam de } \\
\text { mulheres durante } \\
\text { perimenopausa têm uma }\end{array}$ \\
\hline
\end{tabular}

RC: 106172

Disponível em: https://www.nucleodoconhecimento.com.br/saude/reposicao-hormonal 


\begin{tabular}{|c|c|c|}
\hline & & $\begin{array}{l}\text { oportunidade única de diagnosticar } \\
\text { a depressão em seus pacientes e } \\
\text { identificar opções de tratamento } \\
\text { adequadas. }\end{array}$ \\
\hline $\begin{array}{l}\text { Stroke in women - oral } \\
\text { contraception, } \\
\text { pregnancy, and } \\
\text { hormone replacement } \\
\text { therapy }\end{array}$ & $\begin{array}{l}\text { (RANTANEN; } \\
\text { TATLISUMAK., } \\
\text { 2013). } \\
\text { Revisão de } \\
\text { literatura. }\end{array}$ & $\begin{array}{l}\text { As mulheres apresentam fatores } \\
\text { de risco únicos para acidente } \\
\text { vascular cerebral, como } \\
\text { contracepção oral, gravidez, } \\
\text { diminuição do estrogênio após a } \\
\text { menopausa e terapia de reposição } \\
\text { hormonal, que devem ser } \\
\text { avaliados e levados em } \\
\text { consideração nas decisões de } \\
\text { tratamento tanto na fase aguda do } \\
\text { AVC quanto na prevenção } \\
\text { secundária. }\end{array}$ \\
\hline $\begin{array}{l}\text { Fatores psicossociais e } \\
\text { socioeconômicos } \\
\text { relacionados à insônia e } \\
\text { menopausa: Estudo } \\
\text { Pró-Saúde }\end{array}$ & $\begin{array}{l}\text { (ROBAINA et al., } \\
\text { 2015). } \\
\text { Estudo transversal. }\end{array}$ & $\begin{array}{l}\text { A qualidade do sono é um } \\
\text { importante determinante para a } \\
\text { saúde e a qualidade de vida em } \\
\text { geral e, em particular, entre as } \\
\text { mulheres na menopausa. Segundo } \\
\text { Cheng et al., os distúrbios do sono } \\
\text { podem estar relacionados a } \\
\text { questões hormonais, como a falta } \\
\text { de estrógeno. No entanto, os } \\
\text { próprios autores ressaltam que } \\
\text { esses dados não são conclusivos, } \\
\text { já que outros estudos sugerem que } \\
\text { os distúrbios do sono não }\end{array}$ \\
\hline
\end{tabular}




\begin{tabular}{|c|c|c|}
\hline & & $\begin{array}{l}\text { dependem do estado de } \\
\text { menopausa. }\end{array}$ \\
\hline $\begin{array}{l}\text { Menopausal hormone } \\
\text { therapy and breast } \\
\text { cancer risk }\end{array}$ & $\begin{array}{l}\text { (ROZENBERG et } \\
\text { al., 2021). } \\
\text { Revisão narrativa. }\end{array}$ & $\begin{array}{l}\text { Em mulheres na peri e pós- } \\
\text { menopausa com sintomas da } \\
\text { menopausa e/ou fatores de risco } \\
\text { para osteoporose com } \\
\text { necessidade de terapia hormonal } \\
\text { na menopausa, o risco individual } \\
\text { de câncer de mama pode ser } \\
\text { avaliado usando calculadoras } \\
\text { baseadas na Internet. Na maioria } \\
\text { das mulheres, o risco de câncer de } \\
\text { mama em } 5 \text { anos é baixo (<3\%) e } \\
\text { a terapia hormonal na menopausa } \\
\text { é uma opção segura. }\end{array}$ \\
\hline $\begin{array}{lcr}\text { Depoimentos } & \text { de } \\
\text { mulheres sobre a } & \text { e } \\
\text { menopausa e } & 0 \\
\text { tratamento de } & \text { seus } \\
\text { sintomas }\end{array}$ & $\begin{array}{l}\text { (VALADARES et } \\
\text { al., 2008) } \\
\text { Análise secundária } \\
\text { de dados de estudo } \\
\text { populacional de } \\
\text { corte transversal. }\end{array}$ & $\begin{array}{l}\text { Pôde-se constatar, por meio dos } \\
\text { comentários analisados, que as } \\
\text { mulheres, não conseguindo } \\
\text { conviver com os sintomas } \\
\text { climatéricos, fizeram uso da terapia } \\
\text { hormonal, embora inseguras e } \\
\text { superestimando os riscos dessa } \\
\text { terapia. Além disso, sentiam-se } \\
\text { temerosas em relação ao câncer } \\
\text { de mama. Parece existir carência } \\
\text { de conhecimento quanto aos riscos } \\
\text { e benefícios da terapia hormonal, } \\
\text { tanto pelos médicos como pelas } \\
\text { mulheres. }\end{array}$ \\
\hline
\end{tabular}




\begin{tabular}{|c|c|c|}
\hline $\begin{array}{l}\text { Use of hormone } \\
\text { replacement therapy } \\
\text { and risk of breast } \\
\text { cancer: nested case- } \\
\text { control studies using the } \\
\text { QResearch and CPRD } \\
\text { databases. }\end{array}$ & $\begin{array}{l}\text { (VINOGRADOVA et } \\
\text { al., 2020) } \\
\text { Estudo de caso } \\
\text { controle. }\end{array}$ & $\begin{array}{l}\text { Os níveis de risco variaram entre } \\
\text { os tipos de TRH, com maiores } \\
\text { riscos para tratamentos } \\
\text { combinados e para maior duração } \\
\text { de uso }\end{array}$ \\
\hline $\begin{array}{l}\text { Hormone Replacement } \\
\text { Therapy, Breast Cancer }\end{array}$ & $\begin{array}{l}\text { (YOO et al., 2020). } \\
\text { Revisão de }\end{array}$ & $\begin{array}{l}\text { A terapia de reposição hormonal } \\
\text { (TRH) aumenta o risco de câncer }\end{array}$ \\
\hline Risk Factors, and & literatura. & de mama, mas a associação pode \\
\hline $\begin{array}{l}\text { Breast Cancer RISk: A } \\
\text { Nationwide Population- }\end{array}$ & & $\begin{array}{l}\text { variar de acordo com os fatores do } \\
\text { paciente. O risco aumentou }\end{array}$ \\
\hline Based & & $\begin{array}{l}\text { proporcionalmente com a duração } \\
\text { da TRH e diferiu de acordo com o } \\
\text { peso corporal e densidade } \\
\text { mamária. }\end{array}$ \\
\hline
\end{tabular}

Fonte: Próprio autor, 2022.

\subsection{DISCUSSÃO}

Segundo o Instituto Brasileiro de Geografia e Estatística (IBGE), a expectativa de vida da população brasileira feminina aumentou, chegando a 79,4 anos em 2016, com a maior possibilidade de envelhecer, a mulher também começou a vivenciar todas as consequências fisiológicas desse processo, sendo o climatério uma delas. Estima-se que 72 milhões de mulheres no mundo possuem queixas de sintomas moderados a graves decorrentes dessa fase do climatério, sendo que no Brasil há 1,4\% desse total de mulheres e 2 milhões das brasileiras utilizam hormônios exógenos para tratar os 
sintomas do climatério e/ou menopausa, no entanto, o uso indiscriminado destas substâncias pode levar a danos irreversíveis à saúde feminina (SOUZA et al., 2019).

Uma pesquisa que foi aprovada pelo Comitê de Ética em Pesquisa da Faculdade de Medicina de Botucatu-Unesp ressaltou que as usuárias da TRH apresentaram média etária de 50,76 $\pm 3,63$ anos e as não usuárias de 48,95 $\pm 6,27$ anos ( $p=0,014)$. No Brasil, segundo um estudo de base populacional, a prevalência de ondas de calor é de 70,3\% em mulheres no climatério e as variáveis utilizadas que foram: o índice de massa corpórea (IMC), tabagismo, consumo de álcool, escolaridade e antecedente de câncer estão relacionadas com a intensidade das ondas de calor (MIRANDA et al., 2014).

É importante ressaltar que a relação risco $\mathrm{x}$ benefício varia com a idade, da seguinte forma: em mulheres abaixo de 50 anos, normalmente os benefícios superam grandemente os malefícios, devendo a terapia de reposição hormonal (TRH) ser indicada, já em mulheres entre 50 e 60 anos que apresentam sintomas climatéricos, há uma pequena vantagem em relação aos riscos, porém em mulheres acima de 60 anos, os efeitos terapêuticos desejados são equivalentes aos efeitos adversos, e a TRH deve ser criteriosamente acompanhada por um médico, por fim em mulheres acima de 70 anos, os riscos superam os benefícios, sendo fundamental procurar outras alternativas para atenuar os sintomas (POLONINI et al., 2011).

Contudo, é fundamental que haja, nessa fase da vida, um acompanhamento visando à promoção da saúde da mulher, para prevenir possíveis danos. Devendo o médico orientar as mulheres em fase climatérica quanto às alterações fisiológicas e como amenizar tais mudanças para que elas usufruam de uma boa qualidade de vida (FERREIRA et al., 2020; RAGLAN et al., 2019). Almeida et al. (2011) e De Franciscis et al. (2019) ressaltaram que o médico deve instruir a paciente quanto a melhora dos hábitos de vida e quanto ao tipo de reposição hormonal ideal para atenuar o ganho ponderal e a sintomatologia do climatério. Encorajando medidas dietéticas, 
incentivando o exercício físico e regulando o aporte calórico às menores necessidades metabólicas nesta fase da vida da mulher.

\section{Sintomas vasomotores}

Estudos publicados entre 2003 e 2021 nas bases de dados escolhidas salientam de forma unânime a melhora dos sintomas vasomotores com o uso da terapia hormonal. Nessa perspectiva, os fogachos são os sintomas mais inoportunos da menopausa, afetando $60 \%$ a $80 \%$ da população feminina (LANGER et al., 2021).

Para as portadoras das ondas de calor e/ou da sudorese noturna, uma revisão sistemática do Instituto Cochrane relatou uma diminuição de 75\% na frequência e 87\% na severidade dos sintomas vasomotores nas pacientes que utilizaram a terapia de reposição hormonal (PARDINI et al., 2014).

Nos artigos selecionados, as informações publicadas acerca do uso de TRH e fogachos são baseados em doses "standart" de estrógeno (estrógeno conjugado 0,625 mg; $17-\beta$ estradiol oral $1 \mathrm{mg}, 17-\beta$ estradiol transdérmico $50 \mu \mathrm{g} / \mathrm{d}$ ). Todavia, alguns estudos sugerem que baixas doses de estrógeno também são efetivas para alívio dos sintomas vasomotores (PARDINI et al., 2014).

Sintomas urogenitais

É importante ressaltar que os sintomas urogenitais atualmente denominados síndrome geniturinária da menopausa (GSM), são observados em 50\% das mulheres na pós-menopausa e afetam negativamente a qualidade de vida e o bem-estar das pacientes. Pois tais sintomas repercutem em uma bexiga hiperativa, incontinência urinária, falta de lubrificação vaginal, infecção recorrente do trato urinário, atrofia vaginal e de caracteres sexuais secundários. Nessa perspectiva, a $\mathrm{TRH}$, principalmente, pela ação do estrogênio continua sendo o tratamento mais eficaz para o alívio dos sintomas urogenitais (PACIUC, 2020).

Diabetes e intolerância a hidratos de carbono

$\mathrm{RC}: 106172$

Disponível em: https://www.nucleodoconhecimento.com.br/saude/reposicao-hormonal 
O risco aumentado do desenvolvimento de diabetes melito do tipo II em mulheres na menopausa, por um mecanismo ainda não inteiramente delineado, pode se dá pela produção deficitária de estrógenos em decorrência do envelhecimento além do estilo de vida da paciente (PARDINI et al., 2014).

Por ação direta no pâncreas ou nos músculos, observou-se a redução do acúmulo de gordura visceral e melhora da sensibilidade à insulina em pacientes na menopausa e/ou no climatério que fizeram uso da monoterapia com estrogênio ou em associação com a progesterona, o que sinaliza uma diminuição no risco de desenvolvimento do diabetes melito do tipo II (PARDINI et al., 2014).

\section{Osteoporose}

A osteoporose é uma alteração do metabolismo ósseo em que há perda de massa óssea, diminuição da densidade do tecido ósseo, mudanças na microarquitetura histológica do osso e modificações da constituição óssea, que seria uma parte inorgânica e outra orgânica. A primeira é formada por íons de fosfato e cálcio que constituem os cristais de hidroxiapatita. E a segunda é constituída por colágeno tipo I, glicosaminoglicanos, proteoglicanos e glicoproteínas adesivas (ALVES et al., 2021; SOUZA et al., 2019).

Consequentemente, na osteoporose ocorre: rarefação óssea, redução das fibras colágenas, desmineralização e desarranjo da matriz do tecido ósseo, acréscimo na quantidade de osteoclastos e uma redução drástica no número de osteoblastos. Nessa conjuntura, é comum que no climatério as mulheres sofram com o hipoestrogenismo que está intrínseco ao mecanismo de aumento da reabsorção óssea por maior atividade dos osteoclastos, engendrando a perda de massa óssea, relacionada à osteoporose, e maior frequência de fraturas vertebrais, radiais e femorais (ANAGNOSTIS et al., 2020; ALVES et al., 2021).

Além do aludido, Alves et al. (2021) constatou em seu trabalho a importância da atividade física e do estrogênio para prevenir a osteoporose. A qual é uma doença 
crônica e degenerativa frequente em mulheres no climatério e/ou menopausa. Anagnostis et al. (2020) e Gosset et al. (2021) discorreram também em seus artigos sobre a eficácia da TRH na prevenção da perda óssea relacionada à menopausa e na diminuição do risco de fratura.

Nesse contexto, a terapia de reposição hormonal (estrogênio isolado ou associado a progestágenos) age diretamente no osso evitando a reabsorção e, indiretamente na ação do paratormônio e da calcitonina, aumentando a absorção intestinal e a conservação renal do cálcio (GOSSET et al., 2021). Dessa forma, a reposição hormonal no climatério age evitando a perda óssea, aumentando a densidade mineral do tecido ósseo, reduzindo o risco de fraturas de quadril e de coluna, melhorando a microarquitetura histológica do osso, conservando as partes orgânica e inorgânica do osso, aumentando o número de osteoblastos, osteócitos e osteoprotegerina (SOUZA et al., 2019; ALVES et al., 2021).

Portanto, na ausência de contraindicação, o uso de TRH deve ser considerado como a $1^{\underline{a}}$ opção para a manutenção da microarquitetura óssea naquelas mulheres em que não se justificam medicamentos ativos específicos para o tecido ósseo (GOSSET et al., 2021).

Doenças cardiovasculares

A maioria dos estudos selecionados evidenciaram que a terapia hormonal no climatério tem efeito cardioprotetor por alterar os níveis de HDL, LDL, colesterol total, apo-B, fibrinogênio e retarda os estágios iniciais de aterosclerose nas pacientes em uso contínuo e adequado da terapêutica (PALMISANO et al., 2017; NAFTOLIN et al., 2019). A TRH também atua na estabilização das placas ateroscleróticas, na redução da espessura da camada íntima-média da carótida e na diminuição dos escores de cálcio da artéria coronária (GAMBACCIANI et al., 2019; NAFTOLIN et al., 2019).

Dessa forma, estes achados suportam a tese de que a adesão à TRH diminui o risco de doenças coronarianas em mulheres (POLONINI et al., 2011). Além do aludido, 
outro efeito benéfico é a vasodilatação pela liberação de óxido nítrico pelas células endoteliais gerando uma redução na progressão da musculatura lisa vascular que auxilia na redução da pressão arterial (SOUZA et al., 2019). O estudo prospectivo randomizado Women's Health Initiative (WHI) e o artigo Early Versus Late Intervention Trial (ELITE) evidenciaram que começar TRH dentro de 5 a 10 anos da menopausa é fundamental para o sucesso da cardioproteção (NAFTOLIN et al., 2019).

Saúde mental

Fernandes e Rozenthal. (2008) analisaram o quanto a saúde mental pode ser fragilizada pelas alterações hormonais do climatério. Tal análise foi realizada por meio de estudo transversal com 151 mulheres entre 40 e 65 anos de idade, usuárias de serviço de ginecologia geral em unidade de atenção básica à saúde no Rio de Janeiro. Ferreira et al. (2013) também salientou em seu estudo a relação da menopausa e/ou climatério com as alterações biopsicossociais engendradas por esta fase da vida feminina.

O climatério constitui-se como um demarcador biopsicossocial do envelhecimento. O qual é vivenciado pelas mulheres através da mudança corporal, fogachos, sudorese noturna, dificuldades sexuais e significantes psicossociais. Tais como, por exemplo, a depressão, a ansiedade, a insônia e o olhar diferenciado da cultura em relação à mulher climatérica que geram um turbilhão de novos sentidos que precisam ser ressignificados (FERREIRA, 2013).

De acordo com um estudo realizado por ROBAINA et al., 2015, a menopausa não recente associou-se com dificuldade de iniciar o sono, de manter o sono e queixa de insônia. Além disso, é importante ressaltar que a relação entre problemas com o sono e fatores psicossociais pode ser bidirecional, sendo que, a insônia pode ser uma consequência de transtornos mentais comuns, da depressão e de distúrbios de ansiedade, mas também pode ser um fator de risco para tais agravos. 
A reposição endócrina pode diminuir a incidência e a prevalência de tais sintomas por agir diretamente no equilíbrio hormonal e de modo indireto, melhorando o físico da paciente, aumentando a autoestima, diminuindo a sarcopenia e a deterioração do corpo humano pela senescência (MARTINS et al., 2009; MIRANDA et al., 2014). Permitindo, dessa maneira, que a mulher tenha uma saúde mental de qualidade, bemestar físico, disposição e vigor em suas atividades (FERNANDES; ROZENTHAL, 2008).

Doença tromboembólica

Os artigos analisados nesta revisão corroboraram com a ideia de que a terapia hormonal no climatério repercute em um aumento significativo no risco de trombose venosa e embolia. Tal risco, certamente, está envolvido com o tipo de estrógeno usado, visto que o uso de estrógenos equinos conjugados aumenta a resistência à proteína C ativada e a produção de trombina (SOUZA et al., 2019; OLIVEIRA et al., 2019).

Atuais estudos observacionais têm demonstrado provável elevação no risco de tromboembolismo venoso com o uso da terapia de reposição hormonal no climatério. Indicando à suspeita de que estrogênios exógenos, como os usados na $T R H$, poderiam aumentar o risco de tromboembolismo, podendo ser elevado em mulheres com história pessoal ou familiar de trombose venosa profunda ou embolismo pulmonar, assim como a existência de graves varicosidades em membros inferiores, obesidade, trauma, cirurgia, câncer, ou permanência no leito por longo período (CAMPIOLO; MEDEIROS; 2003).

Diante das evidências clínicas e epidemiológicas encontradas, é possível destacar que o uso da TRH aumenta entre 2,1 e 3,5 vezes o risco relativo de tromboembolismo venoso. No entanto, o risco absoluto mantém-se baixo, entre 14 e 32 mulheres por 100.000/ano. Mesmo não tendo ainda o conhecimento nacional a respeito da prevalência das trombofilias, sua procura antes do início da TRH é necessária para avaliar o custo-benefício (CAMPIOLO; MEDEIROS; 2003).

$\mathrm{RC}: 106172$

Disponível em: https://www.nucleodoconhecimento.com.br/saude/reposicao-hormonal 
Diante desses fatores, é importante que o profissional da saúde analise as reais indicações da terapia em questão e o desejo da mulher para o uso da medicação. Sendo relevante que o médico antes de indicar uma prescrição de TRH realize uma extensa anamnese e levante os principais fatores de risco para tromboembolismo ou as prováveis contraindicações (BORGES; TAMAZATO; FERREIRA; 2015).

Acidente Vascular Encefálico

O Acidente Vascular Encefálico (AVE) é a terceira causa mais frequente de morte em mulheres e um dos principais motivos de incapacidade (RANTANEN; TATLISUMAK., 2013). O AVE pode ser dividido em isquêmico e hemorrágico. O primeiro corresponde a cerca de $85 \%$ dos casos de AVE e ocorre por oclusão parcial ou total do lúmen vascular e a extensão da lesão é proporcional à duração, à gravidade da redução do fluxo e ao tipo etiológico do AVE isquêmico de acordo com a classificação TOAST (Trial of Org 10172 in Acute Stroke Treatment). O segundo ocorre por rompimento vascular e, consequentemente, hemorragia no sistema nervoso central (FERNANDES et al., 2021).

Não obstante, estudos anteriores relataram que a TRH no climatério poderia prevenir o AVE devido a capacidade do estrogênio de exercer ações complexas e diversas em distintos tecidos do corpo humano. Tais estudos defendiam que o estrogênio tinha ação neuroprotetora, vasodilatadora, capacidade de aumentar a reatividade vascular, efeitos antitrombóticos e hipolipemiantes. Todavia, estudos observacionais descritivos e meta-análises recentes não corroboram com essa ideia e comprovam que a TRH aumenta o risco de AVE, principalmente, o isquêmico (BILLECl et al., 2008).

Nesse ínterim, Billeci et al. (2008) e Gambacciani et al. (2019) avaliaram que embora a TRH tenha sido associada à redução do risco de doença cardíaca em estudos observacionais, os resultados sobre o acidente vascular encefálico não são claros. Artigos recentes indicam que o estrogênio regula a produção de fatores derivados do endotélio, óxido nítrico, prostaciclina, tromboxano, endotelina-1, fator hiperpolarizante derivado do endotélio, peptídeos natriuréticos, superóxido radicais e inibidor da via do 
fator tecidual. Por isso, a TRH aumenta a inflamação e o risco de AVE (BILLECl et al., 2008).

Nessa perspectiva, os riscos de AVE pela TRH diferem dependendo do tipo hormonal utilizado, da formulação farmacológica, da dose prescrita, da duração do tratamento, da via de administração e se um progestagênio foi utilizado. Assim, o tratamento deve ser singularizado para maximizar os benefícios e atenuar os riscos. $\mathrm{E}$ a reavaliação periódica das vantagens e desvantagens de continuar ou descontinuar a TRH é fundamental (LANGER et al., 2021; BILLECl et al., 2008).

\section{Câncer de mama}

O câncer de mama é a neoplasia maligna mais frequente no sexo feminino e a TRH aumenta a incidência e a prevalência desta patologia, porque boa parte dessas neoplasias possuem receptores que se ligam aos hormônios estrogênio e/ou progesterona (VINOGRADOVA et al., 2020; YOO et al., 2020).

Na maioria das pacientes, o risco de câncer de mama em 5 anos é baixo $(<3 \%)$ e o TRH é uma opção segura. Todavia, a prescrição da TRH deve ser realizada com cautela em mulheres com risco intermediário (3-6\%) e não deve ser prescrita naquelas com alto risco de câncer de mama $(>6 \%)$.

A TRH somente com estrogênio e a TRH com estrogênio-progesterona contendo progesterona micronizada ou didrogesterona estão relacionadas a menor risco de câncer de mama em comparação com outros regimes de TRH combinado (ROZENBERG et al., 2021). Portanto, o risco de desenvolver câncer de mama depende dos tipos de hormônios utilizados para a reposição, com maiores riscos para tratamentos combinados e para maior tempo de uso (VINOGRADOVA et al., 2020).

Além do exposto, Bezerra et al. (2019) esclareceu que apesar da TRH aumentar o risco de câncer de mama, doença tromboembólica e AVE, tais riscos são baixos no primeiro ano de tratamento. E a TRH é fundamental para tratar e amenizar a 
sintomatologia do climatério, visando beneficiar a qualidade de vida das pacientes que fazem uso da reposição endócrina.

\section{Câncer de ovário}

A TRH está associada aos cânceres de ovário seroso e endometrióide, porém não aos tipos de células mucinosas ou claras (LA VECCHIA, 2017). O mecanismo subjacente à relação da $\mathrm{TRH}$ no climatério com câncer de ovário não é bem elucidado. Uma teoria propõe que os altos níveis de gonadotrofinas no período da menopausa e/ou climatério atuam como promotores nos ovários afetados.

Assim, tais achados implicam que a TRH na menopausa e/ou climatério poderia atenuar o risco de câncer ovariano, amenizando os níveis de gonadotrofinas. Todavia, esses benefícios podem ser superados pelo aumento mitótico de células ovarianas, tal proliferação mitótica é induzida pelo estrogênio. Os receptores de estrogênio e progesterona estão presentes na superfície do ovário normal e boa parte dos tumores do tecido ovariano são receptor de estrogênio-positivo (LA VECCHIA, 2017; LIU et al., 2019).

\section{Câncer de endométrio}

O câncer de endométrio é a sétima neoplasia maligna mais comum do mundo no sexo feminino. O principal fator de risco para a hiperplasia endometrial e evolução do câncer é a exposição crônica a altos níveis de estrogênio sem o contraste da progesterona (ARAÚJO JÚNIOR; ATHANAZIO, 2007)

$\mathrm{Na}$ década de 70 , em que a terapia de reposição hormonal (TRH) era realizada somente com estrogênio, houve aumento da incidência de carcinoma de endométrio nas pacientes que estavam em pós-menopausa. Na década de 90, estudos comprovaram que a progesterona tem efeito antiproliferativo sobre o endométrio e que quando é associada ao estrogênio na $\mathrm{TRH}$, reduz a incidência do carcinoma endometrial.

$\mathrm{RC}: 106172$

Disponível em: https://www.nucleodoconhecimento.com.br/saude/reposicao-hormonal 
Assim, a terapia combinada de estrogênio com progesterona diminui o risco de câncer endometrial e atenua os sintomas do climatério. Porém, a TRH com estrogênio e progesterona não é justificada para a prevenção primária do câncer de endométrio em mulheres não climatéricas, pois há um aumento do risco de outros tumores malignos em mulheres na pós-menopausa (ARAÚJO JÚNIOR; ATHANAZIO., 2007)

\section{O papel do médico em relação às pacientes no climatério}

O papel do médico em relação às pacientes no climatério é de grande valia para que as mulheres possam usufruir de uma consulta qualificada e de uma atenção integral à sua pessoa. Portanto, a prevenção e a promoção da saúde da mulher no período climatérico fazem-se essenciais para a melhora sintomática, psicológica e sexual da mulher (RAGLAN et al., 2019).

Nessa conjuntura, antes de qualquer prescrição é papel do médico saber quando contraindicar a TRH. Existem situações, embora ínfimas, em que a terapia de reposição hormonal deve ser desaconselhada. Pode-se dividir as contraindicações ao uso da TRH em absolutas (câncer de mama, doença hepática grave, câncer de endométrio, doença tromboembólica aguda e cardiovascular, sangramento vaginal não esclarecido e porfiria), e relativas (hipertensão arterial não controlada, diabetes mellitus não controlada, endometriose e miomatose uterina) (PARDINI et al., 2014).

Quanto à doença cardiovascular, o consenso da Sociedade Internacional de Menopausa ressaltou que a TRH não está contraindicada a mulheres hipertensas e, em certos casos, a TRH pode diminuir a pressão arterial sistêmica. Em contrapartida, a TRH deve ser contraindicada para mulheres com histórico de infarto do miocárdio, acidente vascular cerebral e embolia pulmonar (PARDINI et al., 2014).

Destarte, após avaliar a possibilidade da prescrição da TRH o médico deve ter perspicácia quanto a melhor via de administração, a classificação dos estrógenos (sintéticos ou naturais), a associação do progestágeno ao estrógeno, a adição da reposição androgênica ao esquema terapêutico e a dose apropriada da TRH para 
cada paciente. Haja vista que o esquema terapêutico deve ser adequado ao perfil da paciente (PARDINI et al., 2014).

Pacientes com hipercolesterolemia podem ser beneficiadas pelo uso oral da $\mathrm{TRH}$, pois esta via diminui o LDL (Low Density Lipoproteins ou Lipoproteínas de baixa densidade) e aumenta o HDL (High Density Lipoproteins ou Lipoproteínas de alta densidade). Em outra ótica, mulheres com hipertrigliceridemia, alto risco de doenças cardiovasculares ou fenômenos tromboembólicos têm vantagens com a administração da TRH por vias não orais. Pois tais vias usufruem de um perfil metabólico neutro e menores estímulos aos fatores de coagulação e às proteínas hepáticas, já que não há metabolismo de primeira passagem pelo fígado (PARDINI et al., 2014).

Dessa forma, o médico deve determinar individualmente o esquema terapêutico e a dose apropriada da TRH para cada paciente com a finalidade de reduzir os efeitos adversos. Tais como retenção de líquidos, êmese, náusea, cefaléia, sensibilidade mamária, inchaço e sangramento vaginal. Assim, o profissional de saúde deve incluir na discussão com suas pacientes todas as abordagens disponíveis para alívio dos sintomas do climatério, concedendo todas as informações necessárias para uma escolha consciente e compartilhada. Tendo como finalidade uma real personalização da abordagem das queixas nesta fase delicada da vida da mulher (DE FRANCISCIS et al., 2019).

Nesse ínterim, a atenção deve ser direcionada, especificamente, ao cuidado continuado. Dessa maneira, o médico deve acolher as pacientes climatéricas, atender de forma humanizada e empática, orientá-las adequadamente, sanar as dúvidas e os medos das pacientes, propor-lhes os meios adequados e seguros para promover seu bem-estar e empoderá-las com conhecimentos que Ihes permitam vivenciar o período com menos repercussões negativas em suas vidas (FERREIRA et al., 2020; RAGLAN et al., 2019). 


\section{CONSIDERAÇÕES FINAIS}

Em suma, respondendo à questão norteadora as vantagens da TRH no climatério são: a melhora dos sintomas vasomotores e urogenitais, a diminuição do risco de diabetes, a eficácia na prevenção da osteoporose, o efeito cardioprotetor e a melhora do perfil lipídico da paciente evitando, desse modo, doenças cardiovasculares, a diminuição do risco de câncer endometrial e atua como promotora de uma saúde mental de qualidade. Por outro lado, as desvantagens desta terapia são: o aumento do risco de doença tromboembólica, de AVE, de câncer de mama e de ovário. Portanto, o papel do médico nesse contexto é acolher, atender de forma humanizada e orientar as pacientes climatéricas. O médico deve ainda definir a melhor abordagem terapêutica, por meio de uma avaliação cautelosa do risco e do benefício da TRH.

\section{REFERÊNCIAS}

ALMEIDA, J. P. M.; CARNIDE, C.; BRANQUINHO, M.; GERALDES, F.; ÁGUAS, F. Impacto da terapia hormonal sobre o peso corpóreo. Revista Brasileira de Ginecologia e Obstetrícia, [S.L.], v. 33, n. 10, p. 310-314. Out. 2011. Disponível em: https://www.scielo.br/pdf/rbgo/v33n10/07.pdf. Acesso em: 11/10/2021.

ALVES, L. F. .; CRUZ, I. L. da .; PIO, J. V. F. .; OLIVEIRA, K. P. de .; KARINA MAGALHÃES DA MATA. Osteoporose: fisiopatologia das alterações histológicas do tecido ósseo e prevenção com atividade física. Revista Multidisciplinar em Saúde, [S. I.], v. 2, n. 4, p. 9, 2021. DOI: 10.51161/rems/2717. Disponível em: https://editoraime.com.br/revistas/index.php/rems/article/view/2717. Acesso em: 19/01/2022.

ANAGNOSTIS, P.; BOSDOU, J. K.; VAITSI, K.; GOULIS, D. G.; LABRINOUDAKI, I. Estrogen and bones after menopause: a reappraisal of data and future perspectives. Hormones Athens Greece, v. 20, n. 1, p. 13-21. 9 de jun. 2020. Disponível em: https://pubmed.ncbi.nlm.nih.gov/32519298/. Acesso em: 11/01/2022. 
ARAÚJO JÚNIOR, N. L. C.; ATHANAZIO, D. A. Terapia de reposição hormonal e o câncer do endométrio. Cadernos de Saúde Pública [online], v. 23, n. 11, p. 26132622. Nov. 2007. Disponível em: https://doi.org/10.1590/S0102-311X2007001100009. Acesso em: 21/01/2022.

BEZERRA, T. A.; LIMA, E. C. de S.; ARAÙJO, A. L.; ROSÁRIO, K. D do. Terapia de reposição hormonal na menopausa. Revista de Iniciação Científica e Extensão, v. 2, n. 4, p. 247-9. Ago. 2019. Disponível em: https://revistasfacesa.senaaires.com.br/index.php/iniciacaocientifica/article/view/265/ 205. Acesso em: 03/07/2021.

BILLECI, A. M.; PACIARONI, M.; CASO, V.; \& AGNELLI, G. Hormone replacement therapy and stroke. Current vascular pharmacology, v. 6, n. 2, p. 112-123. 2008. Disponível em: https://pubmed.ncbi.nlm.nih.gov/18393913/. Acesso em: 20/01/2022.

BORGeS, T. F. C.; TAMAZATO, A. P. da S.; FERREIRA, M. S. C. Terapia com Hormônios Sexuais Femininos e Fenômenos Tromboembólicos: uma Revisão de Literatura. Revista Ciências em Saúde, v. 5, n. 2 , p. 158-168. 2015. Disponível em: 186.225.220.186:7474/ojs/index.php/rcsfmit_zero/article/download/334/256/. Acesso em: 23/01/2022.

CAMPIOLO, Dimas José; de MEDEIROS, Sebastião F. Tromboembolismo venoso e terapia de reposição hormonal da menopausa: uma análise clínico-epidemiológica. Revisões Arq Bras Endocrinol Metab, v. 47, n. 5, p. 534-542. Out 2003. Disponível em: $\quad$ https://www.scielo.br/j/abem/a/4r5W6FtZNZ4pGQVmKDQNVCy/?lang=pt. Acesso em: 23/01/2022.

DE FRANCISCIS, P.; COLACURCI, N.; RIEMMA, G.; CONTE, A.; PITTANA, E.; GUIDA, M.; \& SCHIATTARELLA, A. A Nutraceutical Approach to Menopausal Complaints. Medicina, v. 55, n. 9, p. 544. 2019. Disponível em: https://pubmed.ncbi.nlm.nih.gov/31466381/. Acesso em: 20/01/2022. 
FERNANDES, C. G. C.; FERREIRA, D. D.; FURTADO, D. B. da R.; HARTMANN, J.; WINCKLER, J. L.; MARTINS, M. I. M.; MARRONE, C. P . Independência funcional após acidente vascular cerebral (AVC) isquêmico em relação à fisiopatologia de acordo com TOAST. Revista Brasileira de Neurologia, v. 57, n. 1, p. 13-16. 2021. Disponível em: https://revistas.ufrj.br/index.php/rbn/article/download/43073/pdf. Acesso em: 17/01/2022.

FERNANDES, R. de C. L.; ROZENTHAL, M. Avaliação da sintomatologia depressiva de mulheres no climatério com a escala de rastreamento populacional para depressão CES-D. Revista de Psiquiatria do Rio Grande do Sul, v. 30, n. 3, p. 192 - 200. Dez 2008. Disponível em: http://www.scielo.br/scielo.php?script=sci_arttext\&pid=S0101$81082008000400008 \&$ lang=pt. Acesso em: 11/01/2022.

FERREIRA, V. C.; SILVA, M. R. F da.; MONTOVANI, E. H.; COLARES, L. G.; RIBEIRO, A. A.; STOFEL, N. S. Saúde da Mulher, Gênero, Políticas Públicas e Educação Médica: agravos no contexto de pandemia. Revista Brasileira de Educação Médica, [S.L.], v. 44, n. 1, p. 1-8. Set. 2020. Disponível em: https://www.scielo.br/pdf/rbem/v44s1/1981-5271-rbem-44-s1-e147.pdf. Aceito em: 13/09/2021.

FERREIRA, V. N.; CHINELATO, R. S. C.; CASTRO, M. R.; \& FERREIRA, M. E. C. Menopausa: marco biopsicossocial do envelhecimento feminino. Psicologia \& Sociedade, v. 25, n. 2, p. 410-419. 15 fev. 2013. Disponível em: https://www.scielo.br/pdf/psoc/v25n2/18.pdf. Acesso em: 20/01/2022.

GAMBACCIANI, M.; CAGNACCI, A.; LELLO, S. Hormone replacement therapy and prevention of chronic conditions. Climacteric: the journal of the International Menopause Society, v. 22, n. 3, p. 303-306. 10 de jan. 2019. Disponível em: https://pubmed.ncbi.nlm.nih.gov/30626218/. Acesso em 11/01/2022.

GOSSET, A.; POUILLÈS, J. M.; TRÉMOLLIERES, F. Menopausal hormone therapy for the management of osteoporosis. Best practice \& research. Clinical RC: 106172

Disponível em: https://www.nucleodoconhecimento.com.br/saude/reposicao-hormonal 
endocrinology \& metabolism, v. 35, n. 6, p. 101551.02 de jun. 2021. Disponível em: https://pubmed.ncbi.nlm.nih.gov/34119418/. Acesso em: 11/01/2021.

LANGER, R. D.; HODIS, H. N.; LOBO, R. A.; ALLISON, M. A. Hormone replacement therapy - where are we now? Climacteric : the journal of the International Menopause Society, v. 24, n. 1, p. 3-10. 6 jan. 2021. Disponível em: https://pubmed.ncbi.nlm.nih.gov/33403881/. Acesso em 11/01/2022.

LA VECCHIA, C. Ovarian cancer: epidemiology and risk factors. Eur J Cancer Prev, v. 26, n. 1 , p. 55-62. Jan. 2017. Disponível em: https://pubmed.ncbi.nlm.nih.gov/26731563/. Acesso em:12/01/2022.

LIU, Y.; MA, L.; YANG, X.; BIE, J.; LI, D.; SUN, C.; ZHANG, J.; MENG, Y.; \& LIN, J. Menopausal Hormone Replacement Therapy and the Risk of Ovarian Cancer: A MetaAnalysis. Front Endocrinol, v. 10, n. 1, v. 801. 3 dez. 2019. Disponível em: https://pubmed.ncbi.nlm.nih.gov/31849838/. Acesso em: 12/01/2022.

MARTINS, M. A. D.; NAHAS, E. A. P.; NAHAS-NETO, J.; UEMURA, G.; BUTTROS, D. de A. B.; TRAIMAN, P. Qualidade de vida em mulheres na pós-menopausa, usuárias e não usuárias de terapia hormonal. Revista Brasileira de Ginecologia e Obstetrícia, São Paulo, v. 31, n. 4, p. 196-202. Mar. 2009. Disponível em: https://www.scielo.br/pdf/rbgo/v31n4/07.pdf. Acesso em: 22/11/2021.

MIRANDA, J. S. M.; FERREIRA, M. de L. da S. M.; CORRENTEL, J. E. Qualidade de vida em mulheres no climatério atendidas na Atenção Primária. Revista Brasileira de Enfermagem, v. 67, n. 5, p. 803-809. Set-oct. 2014. Disponível em: https://www.scielo.br/pdf/reben/v67n5/0034-7167-reben-67-05-0803.pdf. Acesso em: 17/07/2021.

NAFTOLIN, F.; FRIEDENTHAL, J.; NACHTIGALL, R.; NACHTIGALL, L. Cardiovascular health and the menopausal woman: the role of estrogen and when to 
begin and end hormone treatment. F1000Research, v. 8, n. 1, p. 1576. 3 de set. 2019. Disponível em: https://pubmed.ncbi.nlm.nih.gov/31543950/. Acesso em: 11/01/2022.

OLIVEIRA, B de.; AZEVÊDO, A. C.; BARRETO, W. J.; FERNANDES, C. Q. B. de A. Terapia de Reposição Hormonal: Uma análise dos benefícios e malefícios no manejo de mulheres climatéricas e menopausadas. Ciência de Envelhecimento Humano. 2019.

Disponível

em: https://editorarealize.com.br/editora/anais/cieh/2019/TRABALHO_EV125_MD1_SA3 _ID2586_10062019212834.pdf. Acesso em: 17/07/2021.

PACIUC, J. Hormone Therapy in Menopause. Advances in experimental medicine and biology, v. 1242, n. , p. 89-120. 14 mai. 2020. Disponível em: https://pubmed.ncbi.nlm.nih.gov/32406030/. Acesso em: 11/01/2022.

PALMISANO, B. T.; ZHU, L.; STAFFORD, J. M. Estrogens in the Regulation of Liver Lipid Metabolism. Advances in experimental medicine and biology, v. 1043, n. 1. p. 227-256. 11 de jan. 2017. Disponível em: https://pubmed.ncbi.nlm.nih.gov/29224098/. Acesso em: 11/01/2022.

PARDINI, D. Terapia de reposição hormonal na menopausa. Arquivos Brasileiros de Endocrinologia \& Metabologia, v. 58, n. 2, p. 172-181. Mar. 2014. Disponível em: https://www.scielo.br/pdf/abem/v58n2/0004-2730-abem-58-2-0172.pdf. Acesso em: 27/11/2021.

POLONINI, H. C.; BRANDÃO, M. A. F.; RAPOSO, N. R. B. A terapia de reposição hormonal e a saúde da mulher no climatério: riscos e benefícios. Revista de atenção primária à saúde, [S.L.], v. 14, n. 3, p. 356-359. Set. 2011. Disponível em: file://C:/Users/Dell/Downloads/14737-Texto\%20do\%20artigo-62484-1-1020111217.pdf. Acesso em: 12/09/2021.

RAGLAN, G. B.; SCHULKIN, J.; MICKS, E. Depression during perimenopause: the role of the obstetrician-gynecologist. Archives of women's mental health, v. 23, n. 
1, p. 1-10. 13 fev. 2019. Disponível em: https://pubmed.ncbi.nlm.nih.gov/30758732/. Acesso em: 12/01/2022.

RANTANEN, K.; \& TATLISUMAK, T. Stroke in women - oral contraception, pregnancy, and hormone replacement therapy. Current vascular pharmacology, v. 11, n. 1, p. 58-73. 2013. Disponível em: https://pubmed.ncbi.nlm.nih.gov/22724472/. Acesso em: 20/01/2022.

ROBAINA, J. R.; LOPES, C. S.; ROTENBERG, L.; FAERSTEIN, E. Fatores psicossociais e socioeconômicos relacionados à insônia e menopausa: Estudo PróSaúde. Cadernos de Saúde Pública, v. 31, n. 3, p. 597-606. Mar. 2015. Disponível em: https://www.scielo.br/scielo.php?pid=S0102311X2015000300597\&script=sci_arttext. Acesso em: 20/01/2022.

ROZENBERG, S.; DI PIETRANTONIO, V.; VANDROMME, J.; GILLES, C. Menopausal hormone therapy and breast cancer risk. Best practice \& research. Clinical endocrinology \& metabolism, n. 35, v. 6, p. 101577. 10 set. 2021. Disponível em: https://pubmed.ncbi.nlm.nih.gov/34535397/. Acesso em: 12/01/2022.

VALADARES, A. L.; PINTO-NETO, A M.; CONDE, D. M.; OSIS, M. J.; SOUSA, M. H de.; COSTA-PAIVA, L. Depoimentos de mulheres sobre a menopausa e o tratamento de seus sintomas. Revista da Associação Médica Brasileira, São Paulo, v. 54, n. 4, p. 299-304. Ago. 2008. Disponível em: https://www.scielo.br/pdf/ramb/v54n4/12.pdf. Acesso em: 26/08/2021.

VINOGRADOVA, Y.; COUPLAND, C.; HIPPISLEY-COX, J. Use of hormone replacement therapy and risk of breast cancer: nested case-control studies using the QResearch and CPRD databases. BMJ, v. 371, n. 1, p. 3873. 28 out. 2020. Disponível em: https://pubmed.ncbi.nlm.nih.gov/33115755/. Acesso em: 12/01/2022.

YOO, T. K.; HAN, K. D.; KIM, D.; AHN, J.; PARK, W. C.; CHAE B. J. Hormone Replacement Therapy, Breast Cancer Risk Factors, and Breast Cancer Risk: A 
Nationwide Population-Based Cohort. Cancer Epidemiol Biomarkers Prev, v. 29, n.

7, p. 1341-1347. 16 abr. 2020. Disponível em:

https://pubmed.ncbi.nlm.nih.gov/32299849/. Acesso em: 12/01/2022.

Enviado: Janeiro, 2022.

Aprovado: Fevereiro, 2022.

RC: 106172

Disponível em: https://www.nucleodoconhecimento.com.br/saude/reposicao-hormonal 